\title{
The relationship between the level of amputation and the type of phantom impressions.
}

\author{
Aneta Pirowska ${ }^{1}$, Tomasz Włoch ${ }^{2,3}$, Roman Nowobilski ${ }^{4}$ \\ 1. Institut Robert Merle d'Aubigné, Artificial Limb Centre (CRA - Centre de Rééducation et d'Appareil- \\ lage), Valenton, France \\ 2. Department of Rehabilitation in Internal Diseases, The University of Physical Education in Krakow, \\ Krakow, Poland \\ 3. The University Hospital, Krakow, Poland \\ 4. Institute of Physiotherapy, Faculty of Health Sciences, Jagiellonian University Collegium Medicum on \\ School of Medicine, Krakow, Poland
}

\section{Keywords}

amputation level, phantom sensation, phantom pain, gender

\begin{abstract}
Introduction: Phantom impressions are divided into phantom sensations and phantom pain. There are many factors that affect the quality and intensity of phantom impressions. These include: preoperative pain in the affected limb, the level of amputation, the height and bilterality of amputation, surgical technique, amputation etiology, stump pain, the state of stump healing, demographic and social factors, psychosocial influences and the level of physical activity.
\end{abstract}

Objective: To evaluate the relationship between the height of limb amputation and the type of phantom sensations and phantom pain.

Material and methods: The study included adults meeting the inclusion criteria after single or multiple-limb amputations. We took into account exclusion criteria. The study involved 67 patients after single or multiplelimb amputations, including 46 men and 21 women. The median age was $48.8 \pm 16.2$ years. The study used an interview questionnaire, numerical scale for assessing phantom pain and stump pain and McGill Pain Questionnaire. The study was conducted in two stages: first - on the day of the initiation of rehabilitation (before putting on the prosthetic appliance) and second - after 5 weeks. The second study additionally included: the stage of prosthesis implantation, the type of prosthesis, the time since putting on the prosthesis, total daily wear time and walking time during which the prosthesis is worn. The first measurement was performed in all 67 patients, while the second in 63 subjects.

Results: The subjects with the vascular and traumatic etiology of amputation predominated in the study sample. Gender did not significantly differentiate the distribution of amputation etiology ( $p>0.05)$. Amputations at the level of the lower leg and thigh predominated in the whole group, constituting groups that were equal in terms of the number of subjects. Gender did not significantly differentiate the sample structure in terms of the level of amputation ( $p>0.05$ ). The majority of women had amputations at the thigh level, while men at the lower leg level. Amputations in the left lower limb definitely dominated both in men and women. Gender did not significantly differentiate the sample structure in terms of limb re-amputation ( $p>0.05)$. 
In measurement 1, there was no relationship between the height of amputation and the type of phantom sensation ( $>0.05$ ). In measurement 2, the relationship between the height of amputation and the type of phantom sensation was significant $(\mathrm{p}<0.05)$. Here, "phantom sensations difficult to describe" clearly predominated $(\mathrm{n}=27)$. In the final measurement, the relationship between the height of amputation and the type of phantom sensation turned out to be significant, still with a clear advantage of "phantom sensations difficult to describe". In women, the most common sensation of the "incomplete, immobile phantom limb" reported in the initial measurement takes in the final measurement the form of the "incomplete, movable phantom limb" equally with "phantom sensations difficult to describe". In measurement 1, women showed a relationship between the height of amputation and the presence of phantom pain $(\mathrm{p}<0.05)$.

Conclusions: The occurrence and severity of phantom impressions is related to the level of limb amputation.

495 words 